\title{
Multisystem Inflammatory Disease with Respiratory Failure in a 7-Year-0ld Boy
}

\author{
Gurpreet Phull'1, Prateek J. Shukla', Dinesh K. Pillai' \\ ${ }^{1}$ Division of Pulmonary and Sleep Medicine, MedStar Georgetown University Hospital, Washington DC, USA \\ ${ }^{2}$ Division of Pulmonary and Sleep Medicine, Children's National Health System, Washington DC, USA \\ Email: gurpreet.phull@gunet.georgetown.edu
}

How to cite this paper: Phull, G., Shukla, P.J. and Pillai, D.K. (2018) Multisystem Inflammatory Disease with Respiratory Failure in a 7-Year-Old Boy. Case Reports in Clinical Medicine, 7, 594-600. https://doi.org/10.4236/crcm.2018.712054

Received: August 30, 2018

Accepted: December 2, 2018

Published: December 5, 2018

Copyright () 2018 by authors and Scientific Research Publishing Inc. This work is licensed under the Creative Commons Attribution International License (CC BY 4.0).

http://creativecommons.org/licenses/by/4.0/

\begin{abstract}
Relapsing polychondritis (RP) is a rare episodic and progressive inflammatory disease of presumed autoimmune etiology. The estimated incidence is 3.5 cases per million. Of these only $10 \%$ affect pediatric populations.
\end{abstract}

\section{Keywords}

Polychondritis, Chronic Atrophic, Meyenburg-Altherr-Uehlinger Syndrome, Atrophic Polychondritis, Cartilaginous Arthritic Deafness Syndrome

\section{Case Description}

A 7-year-old African American boy was seen in the ED with respiratory distress, persistent left foot swelling, inability to bear weight, and worsening malaise for several weeks. Parents report a history of intermittent nasal congestion, noisy breathing with worsening hoarseness in voice, along with bilateral conjunctival injection worsening over two months prior to presentation. An outpatient workup for chronic rhinosinusitis included a CT of sinuses revealing a nasal mass, which was biopsied, and histopathologic results were pending on admission.

He was admitted for respiratory distress (physical exam revealed tachypnea, upper airway congestion, suprasternal retractions) and unexplained left foot swelling. Laboratory findings demonstrated increased inflammatory markers ESR 127 and CRP 21. Chest x-ray performed on presentation was normal. During hospitalization, he developed worsening respiratory distress and stridor requiring supplemental oxygen.

Head/chest CT performed revealed a $5 \mathrm{~mm}$ subglottic airway narrowing with diffuse circumferential thickening of the subglottic airway wall and bronchus intermedius narrowing. Despite nebulized therapies and BiPAP, the patient de- 
veloped respiratory failure ( $\mathrm{ABG}$ pH 7.25, pCO2 57, $\mathrm{PO} 245$ ). Following intubation, direct laryngobronchoscopy (DLB) demonstrated grade 3 subglottic stenosis without granulation tissue or tracheal stenosis.

Flexible bronchoscopy identified severe malacia and thick purulent secretions in the right upper lobe and mild malacia within the left upper and lingual lobes. Sinus CT for chronic congestion as an outpatient had revealed a sinus mass. Biopsy showed elastic cartilage with associated acute inflammation confirming diagnosis of RP. Mycophenolate mofetil and high dose steroids were started with minimal improvement over 2 months. Despite treatment he had three admissions for cyanotic events due to upper airway collapse prompting decision for tracheostomy. Child has been stable over the last four months despite persistently elevated inflammatory markers with tracheostomy collar on humidified room air.

Discussion: Relapsing polychondritis (RP) is a rare multisystem disorder of chondromalacia involving any cartilage with peak incidence between the fourth and sixth decade of life. Involvement of the respiratory tract in RP is a dreaded complication with reported $50 \%$ mortality. It is commonly observed as the disease progresses but seldom seen at the onset. This case illustrates that RP can present at young age/early childhood with respiratory distress as an initial symptom and early diagnosis and treatment is critical for disease prognosis.

\section{Introduction}

Relapsing polychondritis (RP) is an extremely rare disease with estimated incidence of 3.5 cases per million, of which children represent 10\% [1]. First described in 1923, the peak incidence occurs in the $4^{\text {th }}$ to $6^{\text {th }}$ decades of life. RP is a presumed autoimmune, progressive inflammatory disease affecting multiple systems through recurrent inflammation and cartilage damage. As the respiratory tract is commonly involved, life threatening airway complications are concerning. Our patients' young age at onset with quick progression to respiratory involvement, along with time to diagnosis is unique.

\section{Case Presentation}

A 7-year-old African American male presented to the emergency department with worsening respiratory distress alongside inability to bear weight on left leg. Symptoms included general malaise, tactile fevers, intermittent epistaxis, noisy breathing, hoarse voice, and two month history of nasal congestion and worsening conjunctival injection. Outpatient workup for chronic rhinosinusitis included a CT of sinuses revealing a nasal mass, which was biopsied, with histopathology results pending on presentation (Figure 1).

On presentation to the ED, physical examination demonstrated ill appearance, bilateral conjunctiva injection, transmitted upper airway breath sounds, tachypnea, supracostal retractions and left dorsal surface foot swelling. AP/Lateral Chest X-ray on initial presentation demonstrated no abnormal cardiopulmonary 


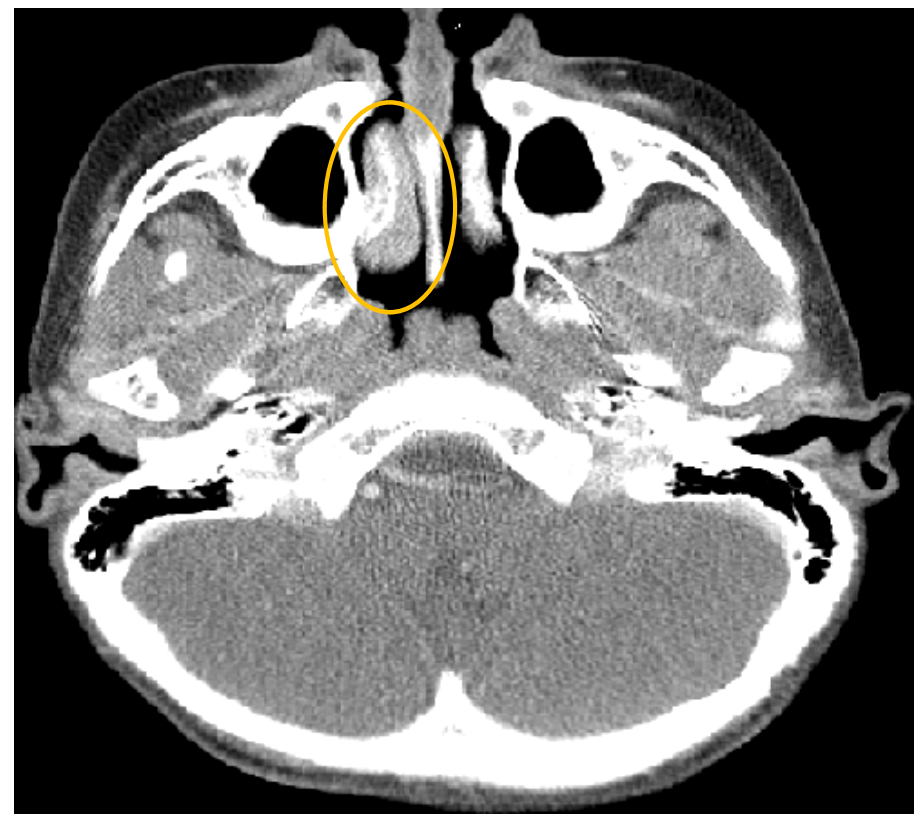

Figure 1. Nasal Septal mass.

findings. MRI of the left knee showed subcutaneous edema and left knee synovitis. Laboratory findings demonstrated increased inflammatory markers ESR 127 and CRP 21. The child was admitted for further workup for respiratory distress and systemic findings.

During his initial hospital stay head/chest CT was performed given worsening stridor, which revealed a $5 \mathrm{~mm}$ subglottic airway narrowing with diffuse circumferential thickening of the subglottic airway wall along with severe bronchus intermedius narrowing (Figure 2).

His respiratory status progressively decompensated, developing respiratory failure, and he was emergently taken to the OR for DLB and intubated. The DLB demonstrated grade 3 subglottic stenosis. Pulmonology was consulted and a flexible bronchoscopy identified severe right upper lobe malacia with thick purulent secretions. The right middle, left upper, and lingula were found to have mild malacia within (Figure 3 ).

During this time, pathologic review of nasal biopsy resulted, identifying elastic cartilage with acute inflammation (Figure 4(a), Figure 4(b)).

The constellation of symptoms including nasal chondritis seen on biopsy, laryngotracheobronchial involvement, and episcleritis seen by opthomologyalong with increased inflammatory markers, was suspicious for rheumatologic disease. Testing including ANA, p-ANCA, c-ANCA, Anti-dsDNA, Anti-type II collagen Abs, IL-1 level, IL-6 level, TNF $\alpha$ level, and antiphospholipid antibodies were performed and negative. Results confirmed chronic arthritis for no other known cause. The constellation of these findings along with histopathology of biopsy and labwork, confirmed the diagnosis of relapsing polychondritis.

Child was started on mycophenalate $500 \mathrm{mg}$ once daily and steroids $20 \mathrm{mg}$ twice daily per rheumatology for multi-system inflammation with respiratory 


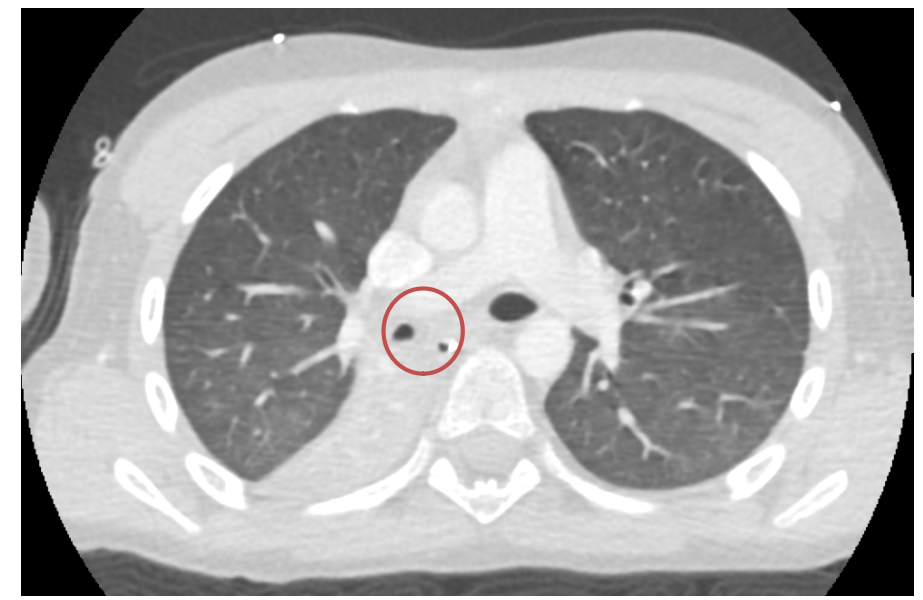

Figure 2. Bronchus intermedius narrowing.

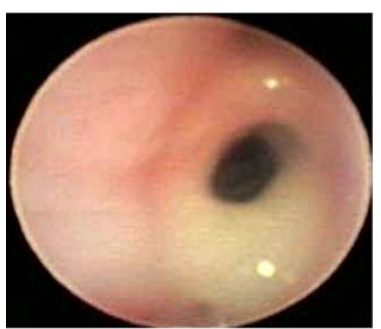

(a)

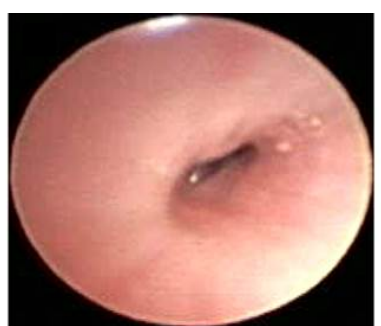

(b)

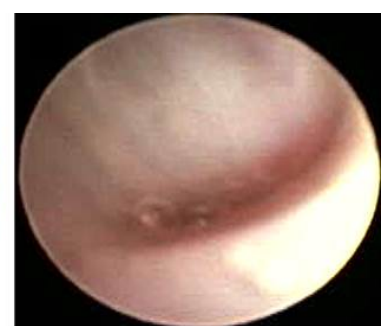

(c)

Figure 3. Flexible bronchoscopy identified severe malacia and thick secretions in the right upper lobe and mild malacia within the left upper lobe and lingula. (a) Thick Secretions; (b) Right upper lobe posterior segment malacia; (c) Apical segment right upper lobe malacia.
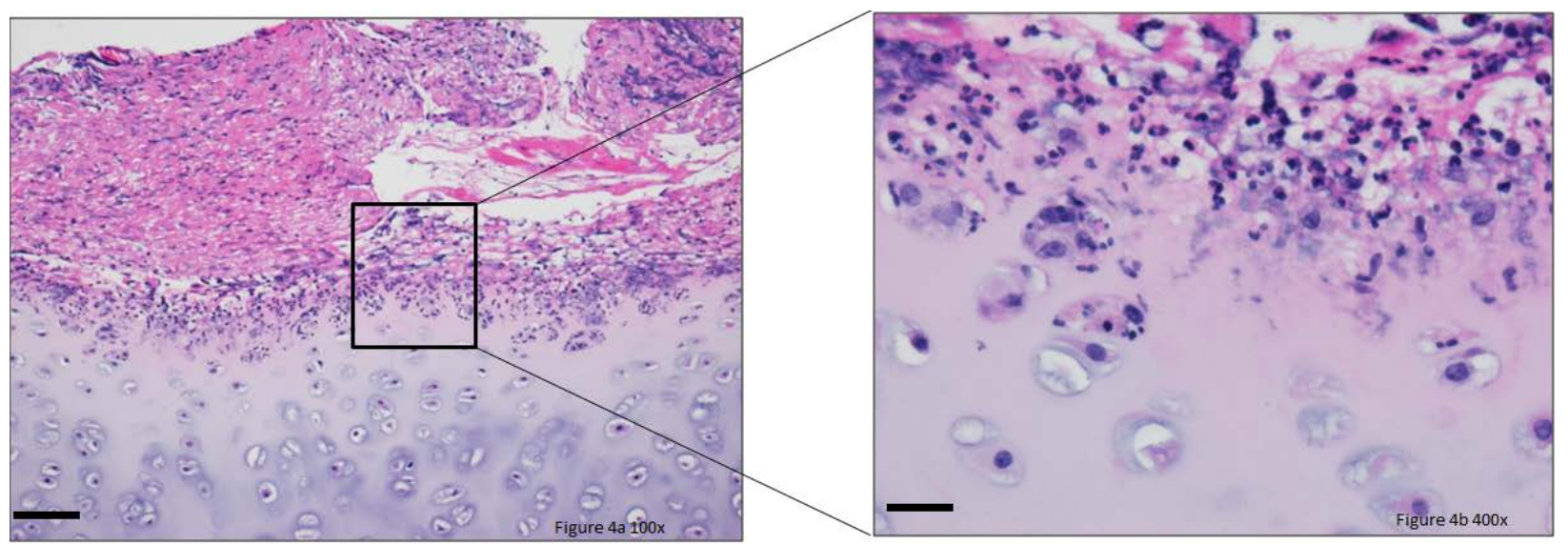

Figure 4. (a) Histopathology of nasal septum, revealing increased inflammatory cells. Hematoxylin and Eosin (H \& E) stain, 100× magnification; (b) Increased inflammatory cells, invading underlying cartilage matrix. Hematoxylin and Eosin (H \& E) stain, $400 \times$ magnification.

tract involvement. He was eventually discharged after extubation and weaning to room air. Within 2 weeks of discharge he re-presented to the ED with respiratory distress felt to be triggered by emotional stressors and was admitted to the PICU for respiratory failure and hypoxemia. Worsening blood gases prompted 
emergent DLB and intubation. He again had subglottic airway narrowing (balloon dilated to $7 \mathrm{~mm}$ ) with proximal and distal tracheal collapse. Flexible bronchoscopy performed demonstrated dynamic malacia and $4+$ Staphylococcus aureus was identified on lavage culture.

He was extubated after initiating therapy (methylprednisone $30 \mathrm{mg} / \mathrm{kg}$, mycophenalate $500 \mathrm{mg}$ twice daily). Following extubation, he was placed on an airway clearance regimen of inhaled ipratropium $(250 \mathrm{mcg})$ and manual chest physical therapy four times a day, and discharged on prednisone $2 \mathrm{mg} / \mathrm{kg} /$ day and mycophenalate $500 \mathrm{mg}$ twice daily.

Within 2 weeks he re-presented to the ED with acutely worsening stridor associated with irritability/anxiety and cyanosis. Symptoms were refractory to racemic epinephrine, albuterol, and supplemental oxygen and he again required intubation. Repeat bronchoscopy revealed generalized subsegmental dynamic bronchomalacia worsening after suctioning. The degree of malacia was less compared to initial bronchoscopy though purulent secretions were greater with growth of $2+$ Moraxella catarrhalis on lavage cultures.

The decision for tracheostomy was reached based on history of multiple intubations secondary to upper airway collapse. Since tracheostomized, he has been stable from a respiratory standpoint on humidified room air, without further hospitalizations despite persistently elevated inflammatory markers. To address ongoing arthritis along with anemia and thrombocytopenia, our rheumatology team has treated with rituximab infusions, bi-weekly solumedrol infusions, low dose prednisone, methotrexate and naproxen.

\section{Discussion}

With lower respiratory tract involvement, mortality in RP increases to nearly $50 \%$. Literature review found increased utilization of tracheostomy in pediatric patients with respiratory involvement [2]. Respiratory chondritis (nasal or laryngotracheobronchial) is often asymptomatic earlier in disease. Patients often have cough, dyspnea, stridor, hoarseness, wheezing, and recurrent infections as the disease progresses [3] [4]. Tracheal tenderness due to laryngotracheal inflammation is suggestive of RP, and laryngotracheal involvement is a significant cause of morbidity and acute or chronic mortality.

The mechanism of airway obstruction in RP initially includes inflammation resulting in airway narrowing in active stages. During progression, there is destruction of the laryngeal, tracheal, and bronchial cartilage with resultant dynamic collapse and formation of fibrous tissue causing cicatricial contraction [5] [6].

McAdam criteria are used for the diagnosis of RP and include at least three of the following: ocular inflammation, nasal chondritis, non-erosive seronegative inflammatory polyarthritis, audiovestibular damage, auricular chondritis or respiratory chondritis [7].

The differential diagnosis for RP is broad and consists largely of rheumatologic conditions such as Granulomatosis with Polyangiitis, Sarcoidosis, Rheu- 
matoid arthritis, Reactive arthritis, Polyarteritisnodosa, and Behçet disease. In this case, the child had ocular inflammation, nasal chondritis, non-erosive inflammatory polyarthritis for no other known cause, and respiratory chondritis.

In patients with RP, respiratory tract radiography should include a frontal chest and lateral view of the neck [8]. High-resolution CT may demonstrate diffuse, smooth thickening of the tracheobronchial wall, causing narrowing and airway deformity. It is also sensitive in evaluating concentric narrowing in lobar and segmental bronchi, and bronchiectasis in segmental and subsegmental bronchi [9]. Bronchoscopy is critical in identifying the site, nature, and severity of airway involvement, and can reveal inflammation of the tracheobronchial tree, with dynamic collapse or narrowing [10]. Ultimately, tracheal collapse and laryngeal strictures often require tracheostomy to maintain airway function [6] [7] [8] [9].

Treatment for RP centers on control of inflammation with immunomodulatory therapy or immunosuppressants. As such, treatment is individualized based on severity and response. Medical management primarily includes NSAIDs and corticosteroids (either burst or maintenance doses). Second line therapies include cyclophosphamide, azathioprine, methotrexate, cyclosporine, colchicine, and chlorambucil [10]. New therapies may include immunomodulators like infliximab, etanercept, adalimumab, and abatacept as methods of better control.

Treatment of symptomatic airway disease includes continuous positive airway pressure (CPAP), bilevel positive airway pressure (BiPAP), or mechanical ventilation when tracheal collapse is present.

\section{Conclusion}

In summary, though rare, pediatric relapsing polychondritis can present as a multisystem disease with airway involvement. McAdam criteria for diagnosis include at least three of the following: ocular inflammation, nasal chondritis, non-erosive seronegative inflammatory polyarthritis, audiovestibular damage, auricular chondritis or respiratory chondritis. The gorals of treatment for RP centers on control of inflammation with immunomodulatory therapy or immunosuppressants. When diagnosing suspected pediatric RP, evaluations should include complete assessment of upper and lower airways with consideration of noninvasive ventilation or tracheostomy to overcome airway obstruction.

\section{Consent}

The family/patient has given informed consent.

\section{Conflicts of Interest}

The authors declare no conflicts of interest.

\section{References}

[1] Kent, P.D., Michet Jr., C.J. and Luthra, H.S. (2004) Relapsing Polychondritis. Cur- 
rent Opinion in Rheumatology, 16, 56-61.

https://doi.org/10.1097/00002281-200401000-00011

[2] Fonseca, A.R., de Oliveira, S.K., Rodrigues, M.C., Aymore, I.L., Domingues, R.C. and Sztajnbok, F.R. (2013) Relapsing Polychondritis in Childhood: Three Case Reports, Comparison with Adulthood Disease and Literature Review. Rheumatology International, 33, 1873-1878. https://doi.org/10.1007/s00296-011-2336-6

[3] Rafeq, S., Trentham, D. and Ernst, A. (2010) Pulmonary Manifestations of Relapsing Polychondritis. Clinics in Chest Medicine, 31, 513-518. https://doi.org/10.1016/j.ccm.2010.04.004

[4] Sharma, A., Gnanapandithan, K., Sharma, K. and Sharma, S. (2013) Relapsing Polychondritis: A Review. Clinical Rheumatology, 32, 1575-1583.

https://doi.org/10.1007/s10067-013-2328-x

[5] Michet Jr., C.J., McKenna, C.H., Luthra, H.S. and O'Fallon, W.M. (1986) Relapsing Polychondritis: Survival and Predictive Role of Early Disease Manifestations. Annals of Internal Medicine, 104, 74-78. https://doi.org/10.7326/0003-4819-104-1-74

[6] Mohsenifar, Z., Tashkin, D.P., Carson, S.A. and Bellamy, P.E. (1982) Pulmonary Function in Patients with Relapsing Polychondritis. Chest, 81, 711-717. https://doi.org/10.1378/chest.81.6.711

[7] McAdam, L.P., O’Hanlan, M.A., Bluestone, R. and Pearson, C.M. (1976) Relapsing Polychondritis: Prospective Study of 23 Patients and a Review of the Literature. Medicine (Baltimore), 55, 193-215. https://doi.org/10.1097/00005792-197605000-00001

[8] Crockford, M.P. and Kerr, I.H. (1988) Relapsing Polychondritis. Clinical Radiology, 39, 386-390. https://doi.org/10.1016/S0009-9260(88)80277-0

[9] Davis, S.D., Berkmen, Y.M. and King, T. (1989) Peripheral Bronchial Involvement in Relapsing Polychondritis: Demonstration by Thin-Section CT. AJR American Journal of Roentgenology, 153, 953-954. https://doi.org/10.2214/ajr.153.5.953

[10] Sarodia, B.D., Dasgupta, A. and Mehta, A.C. (1999) Management of Airway Manifestations of Relapsing Polychondritis: Case Reports and Review of Literature. Chest, 116, 1669-1675. https://doi.org/10.1378/chest.116.6.1669 